\title{
Quasi-periodic oscillations in magnetic Cataclysmic Variables: Results for V834 Cen
}

\author{
Hannes Breytenbach* \\ Astrophysics, Cosmology and Gravity Centre, Department of Astronomy, University of Cape \\ Town, South Africa \\ South African Astronomical Observatory, PO Box 9, Observatory, 7935, South Africa \\ E-mail: hannesesaao.ac.za
}

\section{David A. H. Buckley}

Southern African Large Telescope Foundation, PO Box 9, Observatory, 7935, South Africa South African Astronomical Observatory, PO Box 9, Observatory, 7935, South Africa

E-mail: dibnobesaao.ac.za

\section{Jean-Marc Bonnet-Bidaud}

Service d'Astrophysique-Laboratoire AIM, CEA/DSM/Irfu, 91191 Gif-sur-Yvette, France

E-mail: bonnetbidaudecea.fr

\section{Martine Mouchet}

LUTH-Observatoire de Paris, UMR 8102-CNRS, Université Paris-Diderot, 92190 Meudon, France

E-mail: martine.moucheteobspm. fr

\begin{abstract}
We present the first results from a recent campaign to search for and characterise quasi-periodic oscillations in Polars. A brief review of the current theoretical model is given. Power density spectra and time frequency representations of the rapid variability in the optical light from one of the prototypical Polars, V834 Cen, are presented and discussed. We conclude by discussing the planned future work of this campaign towards advancing the understanding of QPOs in mCVs.
\end{abstract}

The Golden Age of Cataclysmic Variables and Related Objects - III, Golden2015

7-12 September 2015

Palermo, Italy

${ }^{*}$ Speaker. 


\section{Introduction}

Magnetic Cataclysmic Variables (mCVs) are an intriguing class of interacting binaries consisting of a highly magnetized White Dwarf (WD) accreting material from a Roche-lobe filling red-dwarf companion $[1,2]$. A preview of the system's geometry may be sketched by considering the flow of material between the two stars (cf. Figure 1). Material on the surface of the companion is lost through the system's inner Lagrange (L1) point whereafter it may ${ }^{1}$ follow a ballistic trajectory in the orbital plane before encountering the magnetosphere ${ }^{2}$ of the WD primary. Once the (ionized) gaseous material enters the primary's magnetosphere, the Lorentz force on an element of the flow becomes comparable to - and eventually stronger than - the gravitational force, causing the trajectory to divert out of the orbital plane and, roughly speaking, attach on the magnetic field lines of the primary. By the time the infalling material encounters the surface of the WD at the magnetic poles, the flow has become highly supersonic, and the resulting collision is energetic enough to produce the majority of the total luminous radiation emanating from the system. The collimated portion of the flow near the WD surface is known as the accretion column. The transition from a relatively cool, supersonic flow to a extremely hot subsonic flow occurs through a hydrodynamic shock region that sharply decelerates the material, thereby randomizing the velocity field and converting bulk kinetic energy to temperature. It is the post-shock region (or post-shock accretion column (PSAC)), that dominates the radiative output of the system across a wide range of wavelengths. This includes X-ray bremsstrahlung from the hot (10-100keV) gas, infra-red and optical cyclotron emission from fast-moving electrons spiralling along magnetic field lines, as well as UV and soft (20-50eV) X-ray emission from the accretion-heated surface of the WD [4, 5, 6].

A defining feature of the highly magnetic $\mathrm{mCVs}$ is that they emit light that is polarized, both linearly and circularly, on the order of several percent (see for example [8]) - a fact which has led to the sobriquet 'Polar' being adopted for them [9]. The presence of polarized emission is a consequence of the fact that most of the radiation in the system is produced in a region with high magnetic field where charged particles radiate via the cyclotron mechanism. The polarized emission is thus produced by free electrons in the PSAC, and is most prominent in optical and infrared. The prototype, AM Herculis - the first identified polar - was indeed discovered to be an X-ray emitter by the UHURU X-ray satellite, and subsequently identified as compact magnetic accretor from its polarization [10, 11]. Subsequent X-ray surveys by HEAO-1, EXOSAT, ROSAT, RXTE, INTEGRAL, Swift, inter alia, have now brought the number of known Polars to 116 (with 30 more suspected as such) [12].

In general, the accretion flow may be far more complex than the idealised (synchronous, unipolar) scenario described above. In the case of FL Ceti, for example, recent high time resolution photometry with SALTICAM on the South African Large Telescope (SALT) has revealed, through eclipse mapping, unambiguous evidence for two accretion hot-spots on the surface of the WD [13]. The detailed trajectory of the accretion stream is largely determined by the parameters of the WD

\footnotetext{
${ }^{1}$ Here we say "may", since the ballistic inflow might not be present, or might be very brief, if the primary magnetosphere extends as far as L1.

${ }^{2}$ The size of the magnetosphere depends primarily on the magnetic field strength of the WD, which can range from $\sim 10^{7}$ to $\sim 10^{9} \mathrm{G}$ (see [3] for a recent review of magnetic WDs). Formally, we can take the size of the magnetosphere to be specified by the Alfvén radius - the radius at which the magnetic energy density matches the kinetic energy density.
} 


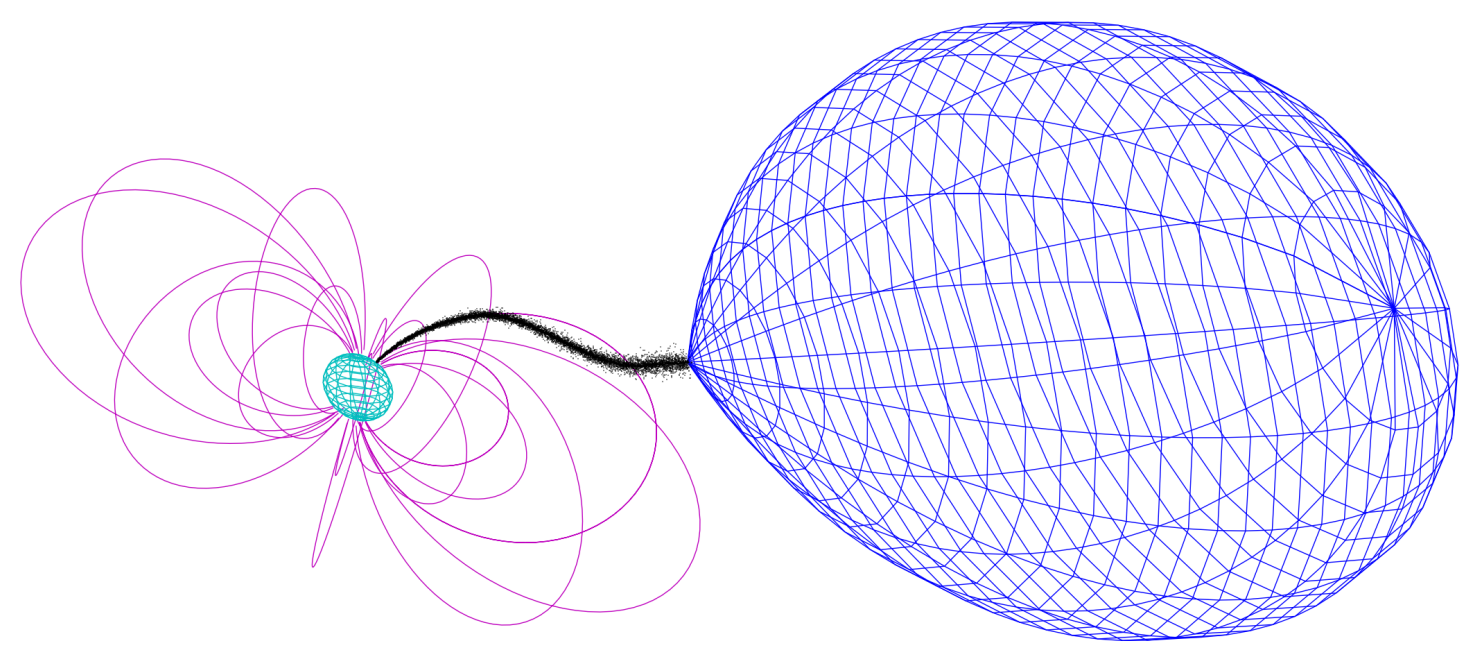

Figure 1: A model representation of a Polar showing: The White Dwarf in cyan with its magnetic field lines in magenta; the Roche lobe of the secondary in blue, and the particle stream between the two in black. For the purpose of illustration, the following system parameters where chosen to produce this figure: $M_{1}=M_{W D}=0.8 M_{\odot}, M_{2}=0.3 M_{\odot}, P_{o r b}=1.5 \mathrm{~h}$. The WD radius was calculated using the Nauenberg mass radius relation [7]. The magnetic field lines displayed are two shells of a dipole field, tilted by $20^{\circ}$ from the orbital axis of the binary. If the binary is synchronised, as is the case for most of the polars, the orientation of the magnetic field with respect to the secondary remains static (in the configuration shown) throughout the orbit.

magnetic field: geometry (dipole or multi-pole), magnetic axis tilt, field centre position (which may not coincide with the WD centre); but also by the accretion rate, WD mass - and spin period. With variation of these parameters, a great variety of accretion geometries become possible (see for e.g. [14]). Such varied scenarios can readily be explored using numerical techniques (see for example [15]). Tantalising evidence for multi-polar accretion geometries has also emerged from spectropolarimetric studies: Employing the Zeeman tomography technique developed in [16], it has been shown that, at least 3 polars (EF Eri, BL Hyi and CP Tuc) possess magnetic field structures significantly more complex than simple offset dipoles [17]. Recent numerical work has taken the cue from such observational findings, and studies that explore the implications of accretion in more complex magnetic geometries have begun to appear [18].

\subsection{Quasi-Periodic Oscillations in the Accretion columns of Polars}

The first detection of quasi-periodic intensity variation in the optical light of polars was accomplished by Middleditch (1982), who described a "noise" feature at $\sim 0.4-0.8 \mathrm{~Hz}(1.25-2.5$ s) with r.m.s. amplitude of a few percent in the systems AN UMa and V834 Cen [19]. This discovery came not long after the prediction by Langer et al. (1981), based on a numerical study of the time-dependent hydrodynamical equations of a 1 dimensional flow, that the PSAC should be thermally unstable [20]. During the intervening 3 decades, numerous studies have expanded on this originative analysis, leading to the emergence of a coherent theoretical model to explain the stability of accretion flows onto compact magnetic objects, and hence, the presence of QPOs 
(see review by $\mathrm{Wu}$ (2000) and references therein)[21]. The stability of the PSAC is determined, primarily, by the cooling processes involved. Flows dominated by bremsstrahlung cooling, tend to be thermally unstable. However, the presence of cooling processes with a stronger temperature dependence (such as cyclotron or Compton cooling), has a stabilising effect.

Intuitively, we can understand how oscillations are generated as follows: Suppose a perturbation in the accretion flow provides a force which pushes the shock front upward with a small non-zero velocity. The overall volume of gas in the PSAC has therefore increased and, under adiabatic expansion, its temperature will tend to decrease. On the other hand, relative to the rest frame of the shock, infalling material is now arriving with an increased velocity, therefore becoming thermalized at a higher temperature, depositing heat into the PSAC. Here we can see how the temperature dependence of the cooling processes play the crucial role in determining if the oscillation will propagate. If the radiative cooling is effective, the newly added hot gas will quickly shed its excess heat as photons and tend to contract, causing the initial perturbation to equilibrate. If, however, cooling is ineffective, the excess heat dumped in the post-shock region will continue driving expansion, contributing to the upward perturbation of the front. We can argue that, once the cooling rate of the post-shock region matches the rate of heat deposition from shocked gas, the shock front will halt, momently beginning to move in the opposite direction as the post-shock gas cools and contracts. The inverse of the above mechanism now applies: as the shock moves downward, infalling gas is now arriving relatively slower, becoming thermalized at a lower temperature and not contributing more heat than is being dissipated through cooling. At the bottom end of the stroke, the gas has once more gained enough thermal energy to halt the contraction as the front completes one cycle of the oscillation. ${ }^{3}$ We have seen that, under certain conditions, the PSAC is thermally unstable, giving rise to oscillations in the height of the shock front, the period of which is characterised by the cooling timescale of the post-shock gas.

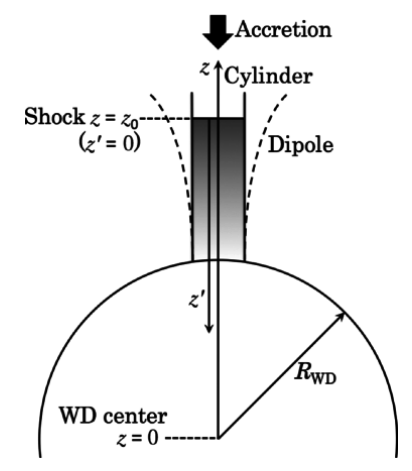

Figure 2: Geometries of the PSAC models. Dashed lines show the dipolar geometry. [Reproduced from [6]].

Although the model just described provides a plausible mechanism for generating QPOs, there are a number of discrepancies, most conspicuously, the absence of X-ray QPOs. An accretion

\footnotetext{
${ }^{3}$ In the preceding discussion, we have strategically avoided a potentially protracted discussion involving a number of factors, such as gravity, radiative forces, opacity, turbulence, etc., all of which may play an important role.
} 
column cooling predominantly through bremsstrahlung, is expected to produce X-rays. A recent comprehensive search for X-ray QPOs in archival XMM-Newton data of 24 Polars (including the 5 that display optical QPOs) has shown this convincingly [22]. It is worth noting, however, that the number of sources in this study constitute only a fraction of the known polars (see below), and as such, conclusions drawn from studying such a sample may be subject to some selection bias.

\section{Observations}

At the time of this writing, there are 5 Polars known to have QPOs. Besides the two systems already mentioned above, QPOs have been detected in optical light from EF Eri, VV Pup, and BL Hyi [23, 24, 25]. All previous detections were made using photomultiplier tubes (PMTs), a technology which has in recent years lost favour against CCD cameras due to the latter's superior sensitivity. Until recently, CCD cameras, despite having a far superior quantum efficiency, have lacked sufficient frame rates to enable studies of short time-scale phenomena such as QPOs. This situation is partly responsible for the nearly 2 decade long dearth in QPO detections in Polars. The recent appearance, however, of fast frame-transfer CCD cameras capable of sub-second integration times (e.g. ULTRACAM [26], or SALTICAM [13]), as well as EM-CCD based cameras (e.g. SHOC [27]) has changed this situation, and has the potential to re-enliven enquiry into the behaviour of $\mathrm{mCV}$ accretion columns, and particularly QPOs.

A systematic program to search for QPOs in Polars, including newly discovered systems, has recently started at SAAO. This program utilizes the Sutherland High-speed Optical Cameras (SHOC) on the SAAO 1.9m reflector [27]. Our eventual aim is to complete a systematic study of the QPO phenomena in a variety of polars with different physical attributes, for example low and high field systems, asynchronous systems, two-pole accretors, low and high mass systems, low and high accretion rate systems. This work will hopefully lead to a better understanding of the physics underlying the QPOs, taking the study from purely phenomenological to one based on quantitative physical parameters. In conjunction with this observational program, we expect that the experimental results from a parallel program of simulating accretion columns in the laboratory will yield further relevant insights [28, 29]. Here we present the first results from this campaign for the Polar V834 Cen.

\section{Results \& Discussion}

Figure 3 shows the differential light curves for V834 Cen on 7 separate nights. The corresponding power density spectra in Figure 4 show that the QPO is almost always detected (the single case here that failed to detect the QPO (Run 2014-07-06) is attributable to bad weather and the short duration of the run (see Table 1). This is encouraging in that it shows that faint, rapid variability can reliably be detected using CCD cameras.

Figure 6 gives an indication of the orbital phase dependence of the QPO. The power excess due to the QPO can be seen in the main panel of the figure as the band of colour $(\sim 0.3-0.8 \mathrm{~Hz})$ stretching across time. Here the intermittent nature of the oscillation is clearly seen: Although there always seems to be some power excess, the total power contained in the QPO, as well as the 


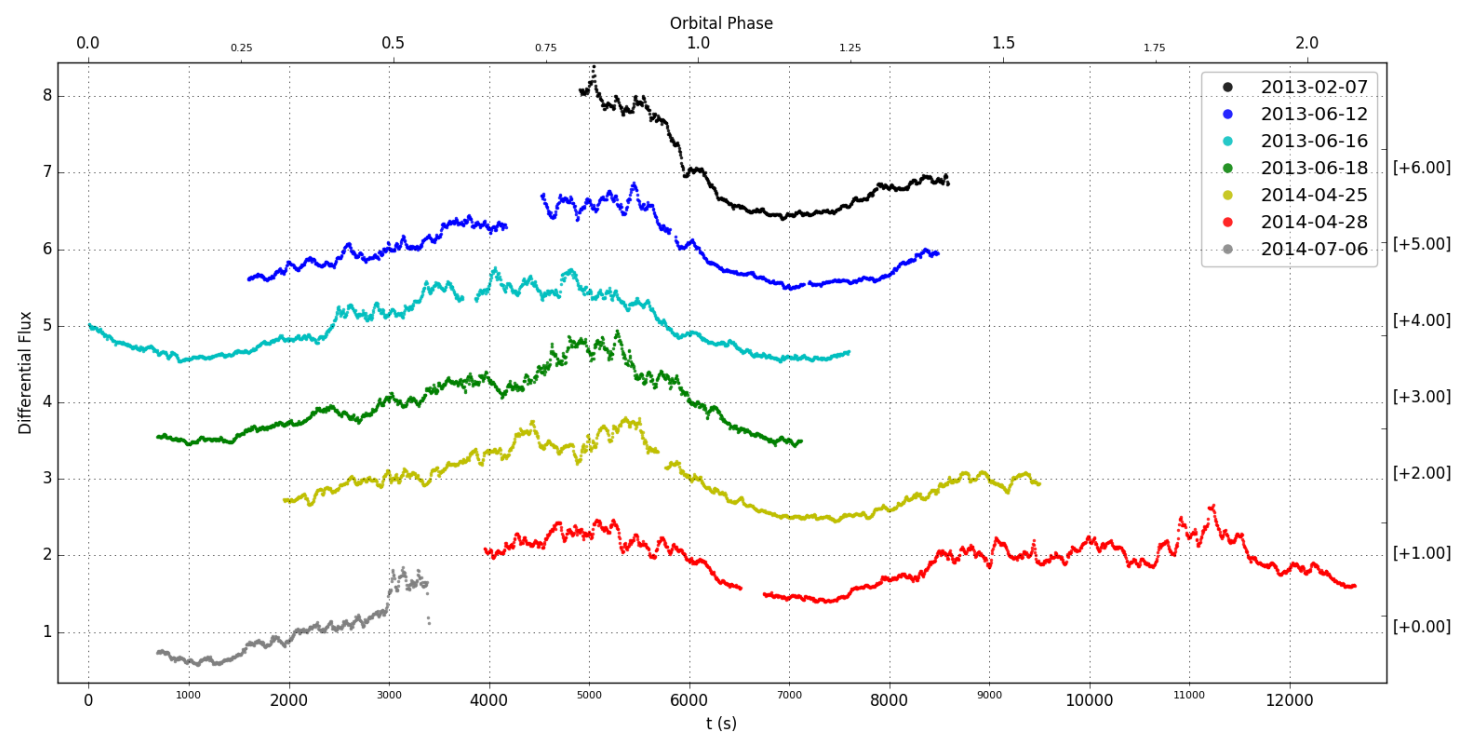

Figure 3: Light curves of V834 Cen for 7 different nights (dates indicated in legend). Displayed are the differential flux values, binned to 5 s intervals. Each light curve is offset for display purposes by the value indicated in the right margin.

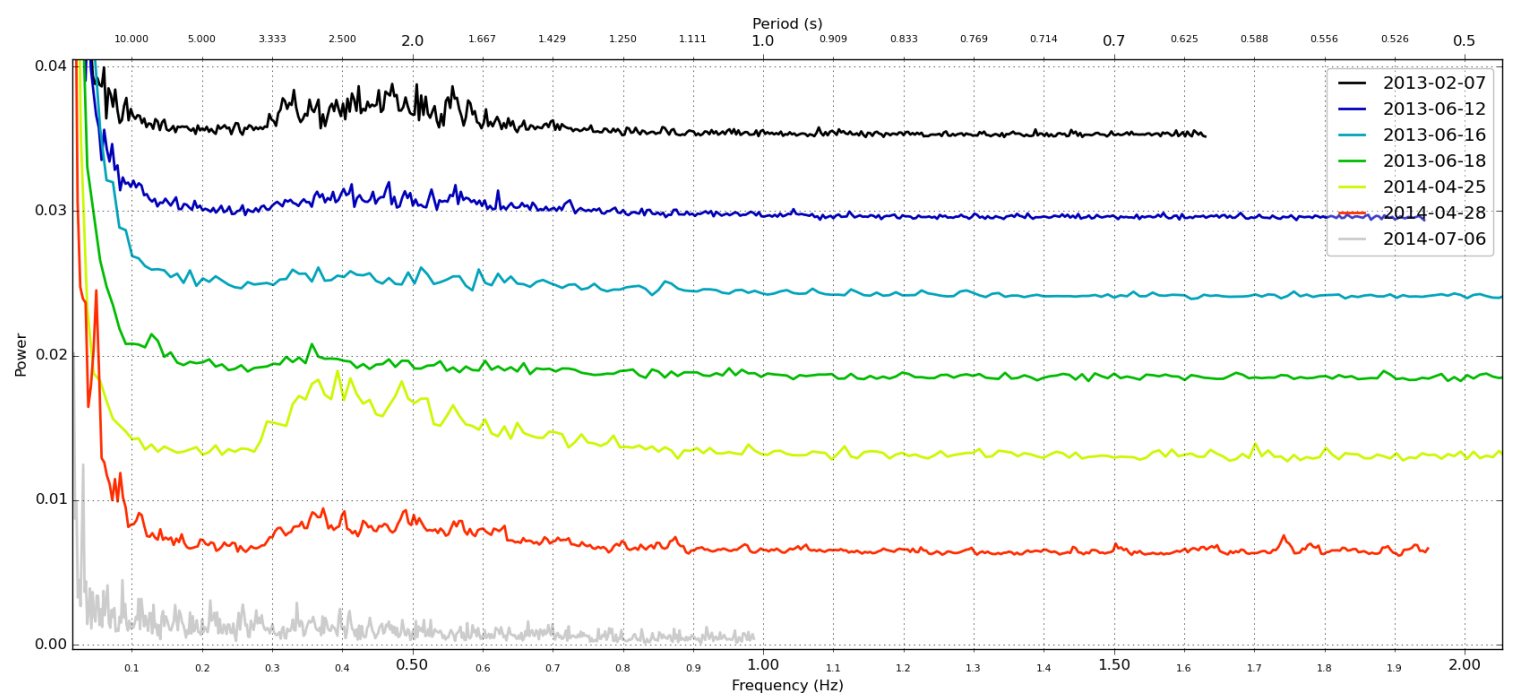

Figure 4: Frequency power spectra of light curves in Figure. 3. The QPO is visible as an excess of power in the range $\sim 0.3-0.8 \mathrm{~Hz}$. The power specturm for each night is offset for display purposes by $0.01 \mathrm{rms}^{2} / \mathrm{Hz}$. 


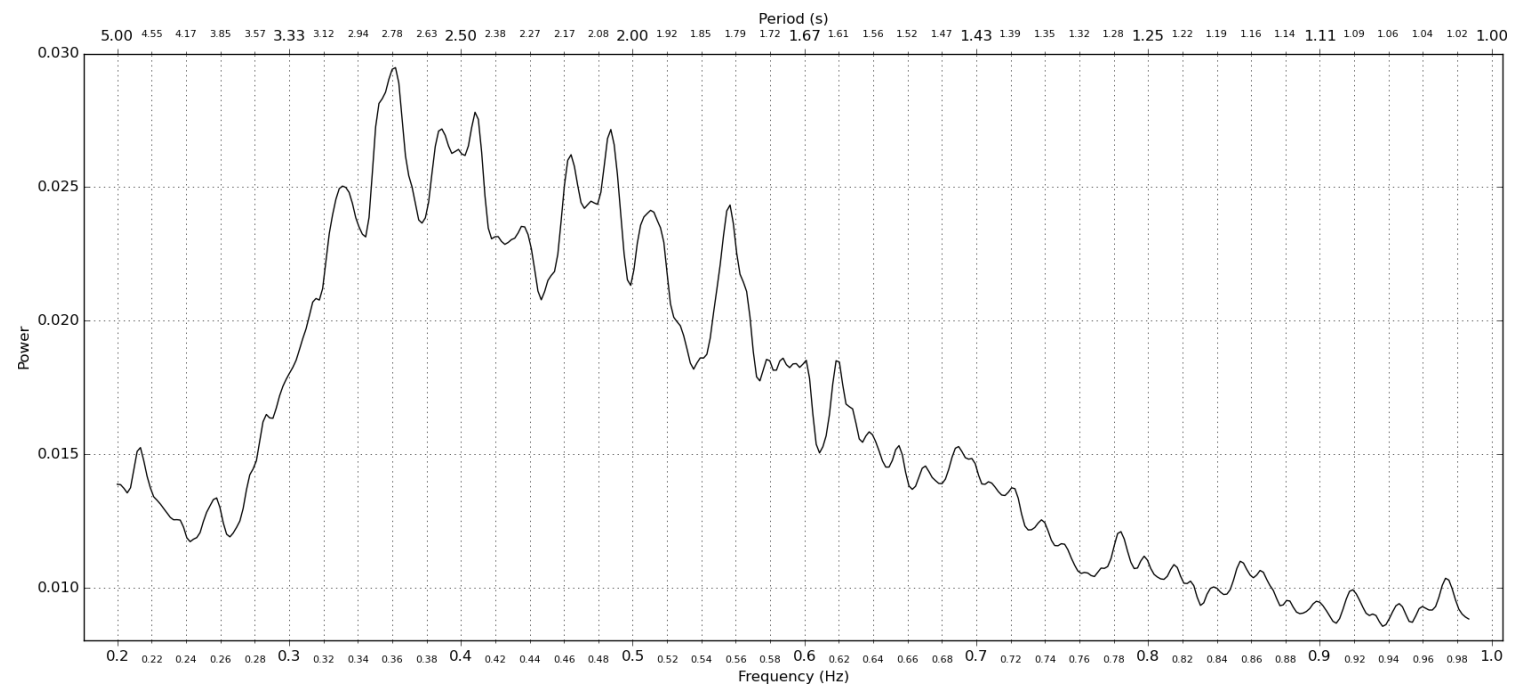

Figure 5: Averaged spectral density estimate of all data presented in Figure 4. Here the QPO appears to be multi-modal: The averaging exposes a number of individual maxima separated by intervening minima. Physical mechanisms that might lead to this kind of behaviour are explored in the text.

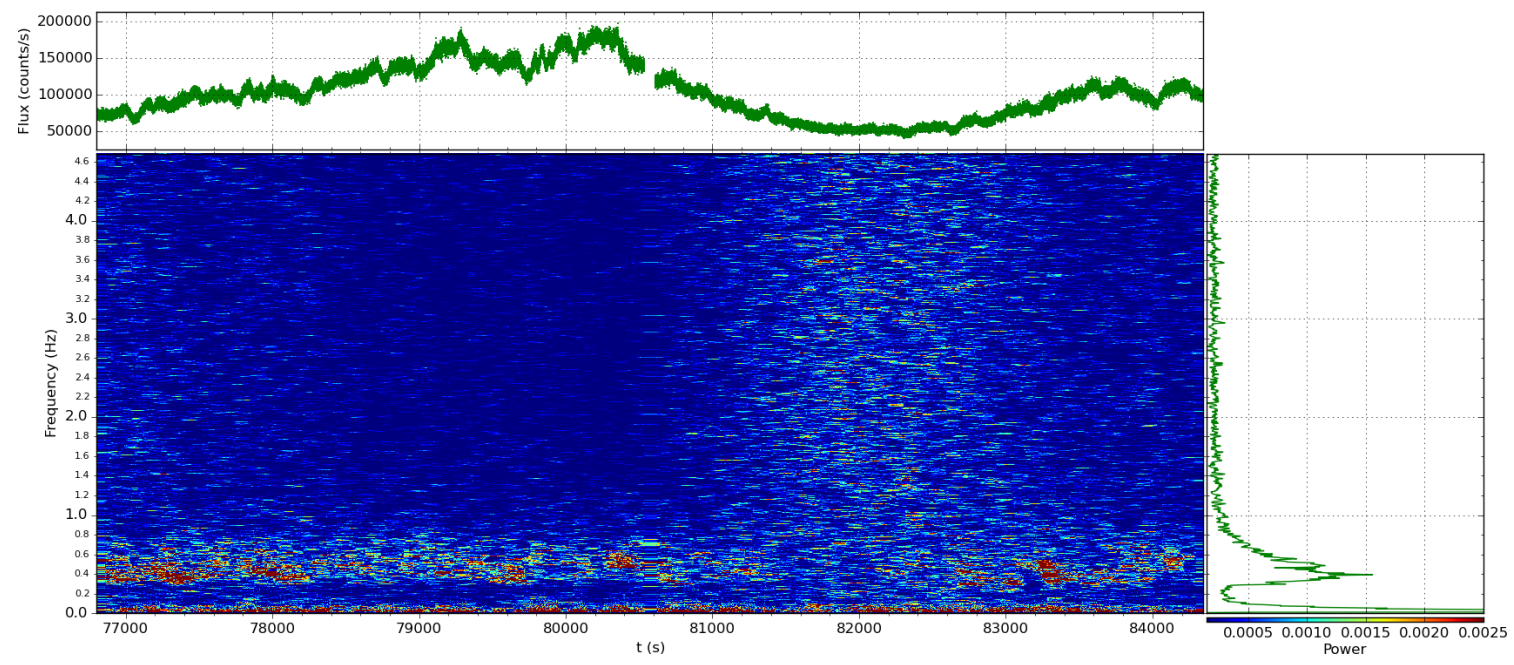

Figure 6: Time Frequency Representation (a.k.a. Dynamic Power Spectrum) for the 2014-04-25 light curve of V834 Cen. The topmost panel shows the light curve covering $\sim 2$ hours $(\sim 1.2$ orbital cycles). The right panel shows the estimated frequency variability spectrum (via Welch's method) for the entire run [30]. The central panel of the figure (mostly blue) is a power density map over the frequency range $0-4.65 \mathrm{~Hz}$. The map was produced by segmenting the light curve into segments of $2^{10}=1024$ data points ( $\sim 100$ s duration), each one overlapping the previous one by $75 \%$, detrending by a cubic polynomial (low pass filtering to suppress red noise) and calculating the Fast Fourier Transform (FFT) periodogram. The power excess from the QPO can be seen in the 0.3 to $0.8 \mathrm{~Hz}$ range $(\sim 1.2-3.3 \mathrm{~s})$. Red noise due to accretion-induced flickering can be seen in both the main- and right panels as a peak in power at lower frequencies. These results are consistent with a previous QPO detection by Larsson (1992)[31]. 


\begin{tabular}{|l|l|l|l|l|}
\hline Run date & Instrument Mode* & EM Gain $\left(e^{-} / A D U\right)$ & Exposure Time (s) & Duration (s) \\
\hline 2013-02-07 & 1 MHz, PreAmp @ 2.5, CON & - & 0.3 & 3681 \\
2013-06-12 & 1 MHz, PreAmp @ 2.4, CON & - & 0.25 & 6909 \\
2013-06-16 & 3 MHz, PreAmp @ 4.9, CON & - & 0.1 & 7599 \\
$2013-06-18$ & 3 MHz, PreAmp @ 4.9, CON & - & 0.1 & 6432 \\
$2014-04-25$ & 1 MHz, PreAmp @ 2.5, EM & 20 & 0.1 & 7546 \\
2014-04-28 & 1 MHz, PreAmp @ 2.5, EM & 12 & 0.1 & 8696 \\
2014-07-06 & 1 MHz, PreAmp @ 2.5, EM & 25 & 0.5 & 2710 \\
\hline
\end{tabular}

* Instrumental Mode is specified as: CCD readout frequency (Pixels/s), pre-amplifier gain in $e^{-} / A D U$, Mode: $\mathrm{CON}=$ Conventional, EM=Electron Multiplying mode. Refer to [27] for details.

Table 1: Observational setup for data presented below.

concentration with frequency tends to vary without any obvious pattern. We note that during the faint phase of the orbit, the QPO falls beneath the detection limit given the uncertainties in the data. The QPO is most strongly detected in the phase where the source is brightening from its faintest (between 83000 and $84000 \mathrm{~s}$ in Figure 6). This pattern appears to be consistent across all the runs.

The multi-modal power distribution seen in Figure 5 and the right panel of Figure 6 deserves some contemplation. One possible explanation that immediately comes to mind is that the individual peaks correspond to harmonics in the oscillation of the shock front. This appears however not to be the case, as the separation in frequency between the peaks is not constant, as one would expect for harmonics. Another possibility is that these correspond to magnetically confined regions within the accretion column that are oscillating (semi-)independently. Having slightly different specific accretion rates, densities etc., each region will oscillate with a slightly different frequency, thus producing a multi-modal power distribution.

\section{Conclusion}

We have presented the first results from a dedicated campaign to search for- and characterise QPOs in Polars. The system V834 Cen has in some ways served as a proof-of-concept for the detection of QPOs using CCD cameras, and the consistency of the results presented with previous studies are positive. On the theoretical front, the standard picture of thermal instability in the accretion column leading to oscillations in the shock height, while providing a mechanism for generating and sustaining QPOs, cannot account for the lack of X-ray QPO detections. Furthermore, the predicted oscillation frequencies for typical WD mass and accretion rate lie somewhere in the range 6-20 Hz[29]. While most observations do not probe this frequency range, we cannot for the moment exclude the possibility of such high frequency oscillations, but note that the current observational data does not agree with this theoretical calculation. As such, we must conclude that the model is still lacking in some key aspects.

In addition to the results presented here, we have obtained high time resolution white-light photometry on a large number of Polars. This data is currently being analysed and will form the 
subject of a forthcoming publication, as well as the first author's $\mathrm{PhD}$ thesis.

\section{Acknowledgements}

This research in supported by the National Research Foundation of South Africa through the Professional Development Programme.

\section{References}

[1] B. Warner. Cataclysmic variable stars, volume 28. Cambridge University Press, 2003.

[2] C. Hellier. Cataclysmic Variable Stars-how and why they vary. Springer Science \& Business Media, 2001.

[3] L. Ferrario, D. de Martino, and B. T. Gänsicke. Magnetic White Dwarfs. Space Science Reviews, pages 1-59, 2015.

[4] D. Q. Lamb and A. R. Masters. $X$ and UV radiation from accreting magnetic degenerate dwarfs. ApJ, 234:L117-L122, 1979. doi:10.1086/183121.

[5] M. König, K. Beuermann, and B. T. Gaensicke. Irradiated atmospheres of accreting magnetic white dwarfs with an application to the polar AM Herculis. A\&A, 449(3):1129-1137, 2006.

[6] T. Hayashi and M. Ishida. A new comprehensive X-ray spectral model from the post-shock accretion column in intermediate polars. MNRAS, 438(3):2267-2277, 2014.

[7] M. Nauenberg. Analytic Approximations to the Mass-Radius Relation and Energy of Zero-Temperature Stars. ApJ, 175:417, July 1972. doi:10.1086/151568.

[8] H. S. Stockman, G. D. Schmidt, G. Berriman, J. Liebert, R. L. Moore, and D. T. Wickramasinghe. A search for circular polarization in cataclysmic variables. ApJ, 401:628-641, December 1992. doi:10.1086/172091.

[9] W. Krzeminski and K. Serkowski. Extremely high circular polarization of an Ursae Majoris. ApJ, 216:L45-L48, August 1977. doi:10.1086/182506.

[10] S. Tapia. Discovery of a magnetic compact star in the AM Herculis/3U 1809+50 system. ApJ, 212:L125-L129, March 1977. doi:10.1086/182390.

[11] A. P. Cowley and D. Crampton. A preliminary model for the X-ray binary AM Herculis. ApJ, 212:L121-L124, March 1977. doi:10.1086/182389.

[12] H. Ritter and U. Kolb. Catalogue of cataclysmic binaries, low-mass X-ray binaries and related objects (Seventh edition). A\&A, 404:301-303, June 2003. doi:10.1051/0004-6361:20030330.

[13] D. O'Donoghue, D. A. H. Buckley, L. A. Balona, D. Bester, L. Botha, J. Brink, D. B. Carter, P. A. Charles, A. Christians, F. Ebrahim, R. Emmerich, W. Esterhuyse, G. P. Evans, C. Fourie, P. Fourie, H. Gajjar, M. Gordon, C. Gumede, M. de Kock, A. Koeslag, W. P. Koorts, H. Kriel, F. Marang, J. G. Meiring, J. W. Menzies, P. Menzies, D. Metcalfe, B. Meyer, L. Nel, J. O’Connor, F. Osman, C. Du Plessis, H. Rall, A. Riddick, E. Romero-Colmenero, S. B. Potter, C. Sass, H. Schalekamp, N. Sessions, S. Siyengo, V. Sopela, H. Steyn, J. Stoffels, J. Scholtz, G. Swart, A. Swat, J. Swiegers, T. Tiheli, P. Vaisanen, W. Whittaker, and F. van Wyk. First science with the Southern African Large Telescope: peering at the accreting polar caps of the eclipsing polar SDSS J015543.40+002807.2. MNRAS, 372:151-162, October 2006. doi:10.1111/j.1365-2966.2006.10834.x. 
[14] A. Norton, O. Butters, T. Parker, and G. Wynn. The accretion flows and evolution of magnetic cataclysmic variables. The Astrophysical Journal, 672(1):524, 2008.

[15] A. G. Zhilkin, D. V. Bisikalo, and P. A. Mason. Full 3D MHD calculations of accretion flow structure in magnetic cataclysmic variables with strong, complex magnetic fields. Astronomy Reports, 56:257-274, April 2012. doi:10.1134/S1063772912040087.

[16] F. Euchner, S. Jordan, K. Beuermann, B. Gänsicke, and F. Hessman. Zeeman tomography of magnetic white dwarfs-I. Reconstruction of the field geometry from synthetic spectra. Astronomy \& Astrophysics, 390(2):633-647, 2002.

[17] K. Beuermann, F. Euchner, K. Reinsch, S. Jordan, and B. T. Gänsicke. Zeeman tomography of magnetic white dwarfs-IV. The complex field structure of the polars EF Eridani, BL Hydri and CP Tucanae. Astronomy \& Astrophysics, 463(2):647-655, 2007.

[18] M. Long, M. M. Romanova, and F. K. Lamb. Accretion onto stars with octupole magnetic fields: Matter flow, hot spots and phase shifts. New Astronomy, 17(2):232 - 245, 2012. ISSN 1384-1076. doi:http://dx.doi.org/10.1016/j.newast.2011.08.001.

[19] J. Middleditch. Two-second variability in AM Herculis binaries. The Astrophysical Journal, 257:L71-L75, 1982.

[20] S. H. Langer, G. Chanmugam, and G. Shaviv. Thermal instability in accretion flows onto degenerate stars. ApJ, 245:L23-L26, April 1981. doi:10.1086/183514.

[21] K. Wu. Accretion onto magnetic white dwarfs. Space Science Reviews, 93(3-4):611-649, 2000.

[22] J. M. Bonnet-Bidaud, M. Mouchet, C. Busschaert, E. Falize, and C. Michaut. Quasi-periodic oscillations in accreting magnetic white dwarfs. I. Observational constraints in X-ray and optical. A\&A, 579:A24, July 2015. doi:10.1051/0004-6361/201425482.

[23] S. Larsson. Discovery of 2-3 S quasi-periodic oscillations in EF ERI. A\&A, 181:L15-L17, July 1987.

[24] S. Larsson. Optical one second quasi-periodic oscillations in VV Puppis. A\&A, 217:146-156, June 1989.

[25] J. Middleditch, J. N. Imamura, and T. Y. Steiman-Cameron. Discovery of Quasi-periodic Oscillations in the AM Herculis Object BL Hydri. ApJ, 489:912-916, November 1997.

[26] V. S. Dhillon, T. R. Marsh, M. J. Stevenson, D. C. Atkinson, P. Kerry, P. T. Peacocke, A. J. A. Vick, S. M. Beard, D. J. Ives, D. W. Lunney, S. A. McLay, C. J. Tierney, J. Kelly, S. P. Littlefair, R. Nicholson, R. Pashley, E. T. Harlaftis, and K. O'Brien. ULTRACAM: an ultrafast, triple-beam CCD camera for high-speed astrophysics. MNRAS, 378:825-840, July 2007. doi:10.1111/j.1365-2966.2007.11881.x.

[27] R. Coppejans, A. A. S. Gulbis, M. M. Kotze, D. L. Coppejans, H. L. Worters, P. A. Woudt, H. Whittal, J. Cloete, and P. Fourie. Characterizing and Commissioning the Sutherland High-Speed Optical Cameras (SHOC). PASP, 125:976-988, August 2013. doi:10.1086/672156.

[28] D. Buckley, S. Potter, E. Kotze, M. Kotze, and H. Breytenbach. New Observations of Accretion Phenomena in Magnetic Cataclysmic Variables. In EPJ Web of Conferences, volume 64, page 07005. EDP Sciences, 2014.

[29] C. Busschaert, É. Falize, C. Michaut, J.-M. Bonnet-Bidaud, and M. Mouchet. Quasi-periodic oscillations in accreting magnetic white dwarfs-II. The asset of numerical modelling for interpreting observations. Astronomy \& Astrophysics, 579:A25, 2015. 
[30] P. D. Welch. The use of fast Fourier transform for the estimation of power spectra: A method based on time averaging over short, modified periodograms. IEEE Transactions on audio and electroacoustics, 15(2):70-73, 1967.

[31] S. Larsson. Rapid optical variability in the AM Herculis object V834 Centauri. I-Quasi-periodic oscillations. Astronomy and Astrophysics, 265:133-143, 1992. 